\title{
fMRI Repetition Suppression for Familiar But Not Arbitrary Actions with Tools
}

\author{
Kenneth F. Valyear, ${ }^{1}$ Jason P. Gallivan, ${ }^{2}$ D. Adam McLean, ${ }^{3}$ and Jody C. Culham ${ }^{3,4}$ \\ ${ }^{1}$ Department of Psychological Sciences, University of Missouri, Columbia, Missouri 65211, ${ }^{2}$ Centre for Neuroscience Studies, Queen's University, Kingston, \\ Ontario, Canada, K7L 3N6, ${ }^{3}$ Centre for Brain and Mind, Natural Sciences Centre and ${ }^{4}$ Department of Psychology, University of Western Ontario, London, \\ Ontario, Canada N6A 3K7
}

For humans, daily life is characterized by routine interaction with many different tools for which corresponding actions are specified and performed according to well-learned procedures. The current study used functional MRI (fMRI) repetition suppression (RS) to identify brain areas underlying the transformation of visually defined tool properties to corresponding motor programs for conventional use. Before grasping and demonstrating how to use a specific tool, participants passively viewed either the same (repeated) tool or a different (non-repeated) tool. Repetition of tools led to reduced fMRI signals (RS) within a selective network of parietal and premotor areas. Comparison with newly learned, arbitrarily defined control actions revealed specificity of RS for tool use, thought to reflect differences in the extent of previous sensorimotor experience. The findings indicate that familiar tools are visually represented within the same sensorimotor areas underlying their dexterous use according to learned properties defined by previous experience. This interpretation resonates with the broader concept of affordance specification considered fundamental to action planning and execution whereby action-relevant object properties (affordances) are visually represented in sensorimotor areas. The current findings extend this view to reveal that affordance specification in humans includes learned object properties defined by previous sensorimotor experience. From an evolutionary perspective, the neural mechanisms identified in the current study offer clear survival advantage, providing fast efficient transformation of visual information to appropriate motor responses based on previous experience.

\section{Introduction}

Contrary to classic views of the motor system, evidence from neural recording studies conducted with nonhuman primates indicates that brain areas responsible for the control of actions specify visual stimuli according to their potential for action in confluence with higher-order goals and intentions (Cisek and Kalaska, 2010). The process whereby action-relevant object properties (affordances) are visually represented in sensorimotor areas is known as affordance specification, considered fundamental to action planning and execution. With grasping, visual object properties register with corresponding motor representations; object size, shape, and orientation activate parietofrontal neurons coding these features for the purpose of controlling the hand accordingly (Taira et al., 1990; Baumann et al., 2009). Multiple lines of evidence suggest that similar neural mechanisms underlying affordance specification exist in humans, including evidence derived from neuroimaging (Grèzes et al., 2003), neuropsychology (Riddoch et al., 1998), and behavioral studies with neurologically healthy individuals (Tucker and Ellis, 1998).

Received Oct. 18, 2011; revised Dec. 9, 2011; accepted Jan. 12, 2012.

Author contributions: K.F.V., J.P.G., D.A.M., and J.C.C. designed research; K.F.V. and D.A.M. performed research; K.F.V. analyzed data; K.F.V., J.P.G., and J.C.C. wrote the paper.

This work was funded by Canadian Institutes of Health Research Grant MOP84293 (J.C.C.) and a Canada Graduate Scholarship from the Natural Sciences and Engineering Council of Canada (K.F.V.).

Correspondence should be addressed to Kenneth F. Valyear, Department of Psychological Sciences, $115 \mathrm{Melvin} \mathrm{H}$. Marx Building, University of Missouri, Columbia, M0 65211. E-mail: kfvalyear@gmail.com.

DOI:10.1523/JNEUROSCI.5270-11.2012

Copyright $\odot 2012$ the authors $\quad 0270-6474 / 12 / 324247-13 \$ 15.00 / 0$
More recent evidence suggests that, for humans, the specification of affordances includes learned object properties defined by previous sensorimotor experience. Simply viewing pictures of tools was found to activate left parietofrontal areas putatively important for actual tool use (Chao and Martin, 2000; Valyear et al., 2007) and to affect response times to initiate real actions according to affordances defined by both structural and functional tool properties (Bub et al., 2003; Bub et al., 2008). More recently, it was shown that affordance specification for tools includes information about predicted outcomes of actions in accordance with their conventional use (Masson et al., 2011), and reaction times to pantomime tool use with tools in hand are facilitated when preceded by passive viewing of real tools (Valyear et al., 2011). These findings support the hypothesis that affordance specification in humans includes access to learned functional properties of objects based on previous experience.

The current study aimed to provide novel neural-based support for the above hypothesis using a functional MRI (fMRI) method known as repetition suppression (RS). Passive viewing of the same (repeated) versus different (non-repeated) tools before actual tool-use demonstration resulted in RS measured as reduced fMRI signal within a selective network of parietofrontal areas. Comparison with newly learned control actions revealed specificity of RS for tool use, thought to reflect differences in the extent of previous sensorimotor experience and thus the strengths of associations underlying tool use versus control actions. Good correspondence with previous tool-related neuroimaging studies as well as relevant neuropsychological findings 
A

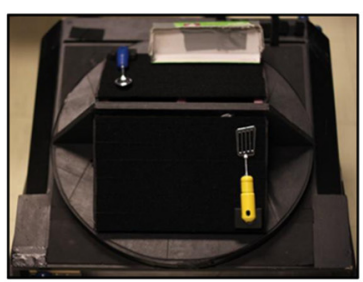

B

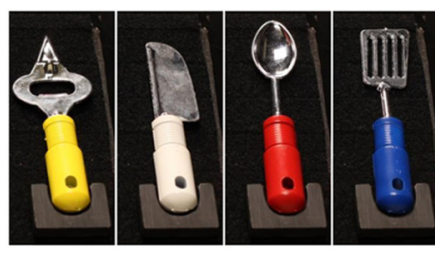

C

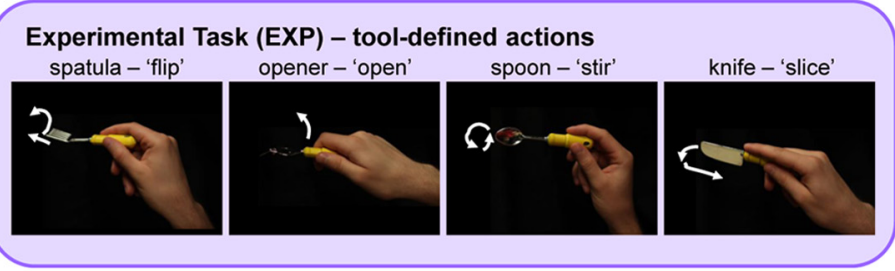

Control Task (CTR) - color-defined actions

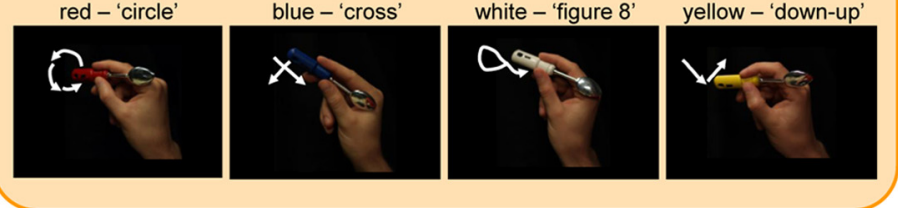

white - 'figure 8'
D

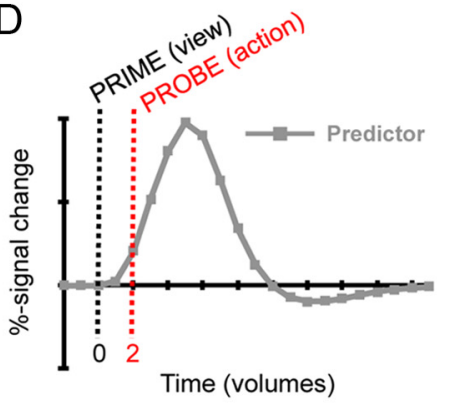

E

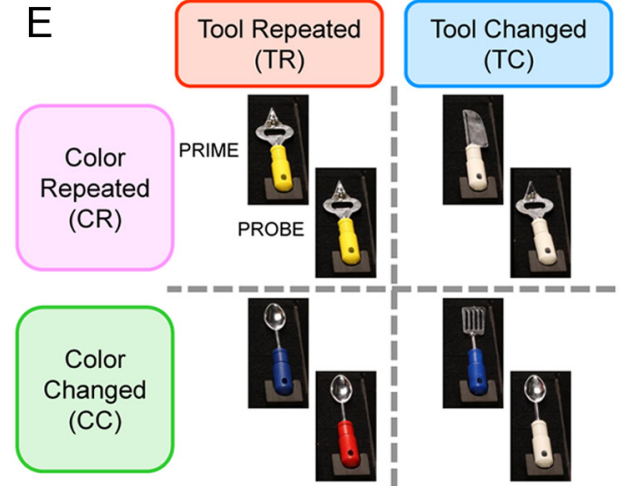

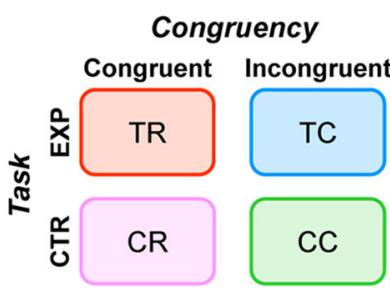

Figure 1. Methods. $A$, An overhead view of the grasping platform and turntable apparatus used to present tools to participants in the scanner. Both presentation sides are visible. The prime side shows a yellow spatula, and the probe side shows a blue spoon in position. On the probe side, a small box was used for participants to place tools in after they had completed their actions. $B$, Examples of tools (bottle opener, knife, spoon, and spatula) and handle colors (yellow, white, red, and blue). C, In the EXP task, participants grasped and used tools according to identity (i.e., the basic movement features of these actions were consistent with those of actual tool use). Actions are shown with arrows drawn to indicate basic movement features. In the (TR task, participants grasped and used tools according to arbitrarily learned associations matching handle color to a specific set of movements. $\boldsymbol{D}$, For each trial, prime and probe events were modeled with a single predictor function aligned to the start of the prime event convolved with Brain Voyager QX two-gamma hemodynamic response function designed to estimate BOLD response characteristics. E, For the EXP task, TR and TC trials were defined as congruent and incongruent, respectively, and RS was predicted to correspond with TC > TR activity (EXP-RS). For the CTR task, CR and CC trials were defined as congruent and incongruent, respectively, and RS was predicted to correspond with $C C>C R$ activity (CTR-RS). Main analysis performed at the group voxelwise level included two independent contrasts, TC > TR to identify EXP-RS, and CC > CR to identify CTR-RS, as well as an additional voxelwise factorial analysis, including task (two levels, EXP and (TR) and congruency (two levels, congruent and incongruent) as factors.

suggest that areas showing RS for tool use correspond with areas important for tool use production. The findings are hypothesized to indicate that tools are visually represented within the same sensorimotor areas underlying their dexterous use according to learned properties defined by previous experience. The results are discussed with respect to previously identified neural mechanisms known as affordance specification whereby action-relevant object properties (affordances) are visually represented in sensorimotor areas. Within this framework, the current findings reveal the importance of previous sensorimotor experience in the specification of object affordances, neural response mechanisms that, from an evolutionary perspective, clearly confer survival advantage.

\section{Materials and Methods}

The current study used fMRI RS to provide novel evidence for the hypothesis that passive viewing of tools involves specification of their properties according to previous sensorimotor experience. RS is characterized by reduced blood oxygenation level-dependent (BOLD) signal associated with repeated versus non-repeated stimulus/task events, commonly interpreted as improved metabolic efficiency of underlying neural processes, although these mechanisms are not yet fully understood and may be attributable to several distinct modifications of underlying cellular events (Grill-Spector et al., 2006). In the main experimental (EXP) task, participants grasped and demonstrated the use of familiar tools (Fig. 1). Action (probe) events were preceded by visual (prime) events involving passive viewing of the same or different tool as that which was then used, comprising tool-repeated (TR) versus tool-changed (TC) trials, respectively. In accordance with the hypothesis that affordance specification of familiar tools includes access to information related to their conventional use, prime events involving passive viewing of tools were predicted to activate corresponding motor programs for conventional use. When actions following prime events involved the same tools, TR trials, (re)activation of similar motor programs was predicted, whereas TC trials were predicted to involve successive activation of distinct motor programs. Repeated versus non-repeated activation of tool-specific motor programs was predicted to correspond with RS, relatively reduced BOLD signal (i.e., TC > TR), within areas underlying tool-use planning and execution.

Notably, the current design differed from most previous fMRI RS studies in that events within a trial involved distinct tasks. Prime events involved viewing tools, whereas probe events involved viewing and acting with tools. From one perspective, inclusion of distinct tasks for prime (visual) and probe (visual and motor) events may be expected to activate distinct brain areas independent of whether or not tools are repeated or changed, and thus the use of the concept of fMRI RS to account for decreased activation for TR versus TC trials may seem inappropriate. However, our main hypothesis specifically predicts that prime events involving passive viewing of tools will activate sensorimotor areas under- 
lying their conventional use. Clearly, probe events involving actual execution of tool use will also activate such areas. It follows that repeated presentation of tools for TR trials is predicted to result in activation of more of the same neural populations relative to TC trials involving distinct tools predicted to activate relatively distinct neural populations. Thus, in the context of our hypothesis, decreased activation for TR versus TC trials is consistent with the concept of fMRI RS, attributable to successive activation of overlapping neural populations. Importantly, our hypothesis specifically implicates parietofrontal areas underlying the sensorimotor control of tool use. It should also be acknowledged that TC $>$ TR activation detected in sensorimotor areas may reflect inhibition of incorrectly activated motor plans by prime events for TC but not TR trials. Critically, either interpretation would provide evidence for the hypothesis that prime events involving passive viewing of tools can activate corresponding motor plans for conventional use.

The novel methodological aspects of the current design, specifically the inclusion of "visual only" followed by "visual and motor" events with tools, are not only uniquely suitable to unequivocally evaluate our main hypothesis but also translate to a major advantage over previous neuroimaging studies of tool use. In the current design, TC and TR conditions ultimately involved the same executed actions so that resultant activations based on their comparison are not attributable to potential motor or somatosensory confounds. Previous imaging studies have used conventional contrast methods to compare tool use with control actions not well equated for kinematic complexity. Resultant activations for tool use may have been attributable to differences in extent, complexity, or duration of movements and/or haptic feedback (for those studies involving actual tool manipulation). Thus, if observed, detection of areas showing TC $>$ TR activity in the current study is expected to provide a more selective account of the neural substrates of tool use than was previously available.

Rather than related to repeated activation of learned motor programs for tool use, decreased activity for TR versus TC trials could in principle reflect correct versus incorrect action cueing independent of learned tool-action associations. If this were the case, other types of congruent versus incongruent stimulus-action associations would be expected to generate RS in the same areas. To test this, we included a control (CTR) task whereby participants grasped the same tools used in the EXP task but, instead of performing actions according to tool identity, actions were performed according to arbitrary associations based on handle color of tools. Trials with repeated handle color, color repeated (CR), would (in theory) signal repeated motor programs, whereas trials with different handle colors, color changed (CC), would signal distinct motor programs. Thus, RS for the CTR task was predicted to correspond with CC $>$ CR activity.

Both EXP and CTR tasks were predicted to show RS in the same brain areas if the effects were attributable to prime stimuli correctly versus incorrectly cuing subsequent actions in general. Alternatively, if RS in the context of the current study reflects the specification of object affordances according to previous sensorimotor experience, then the effects were predicted to be more pronounced for the EXP task and tool-action associations by virtue of their greater experiential strength compared with newly learned color-action associations.

We should clarify from the outset that greater experiential strength with respect to tool-action associations for the EXP versus CTR task is not meant to suggest that participants will have differential experience in the actual performance of either task. Participants will perform both tasks to the same extent (see below, Prescan training). However, if RS in sensorimotor areas underlying tool use was found to be specific to the EXP task, this would be interpreted as related to the fact that previous experience is consistent with the action associations that characterize the EXP but not CTR task. In other words, for the EXP task exclusively, the visuomotor associations that specify the sequence of movements to be performed have the potential to operate in accordance with existing associations defined by previous sensorimotor experience using tools. This is not the case for the newly learned arbitrary visuomotor associations that characterize actions for the CTR task. Note that evidence for such preexisting associations would necessarily correspond with generalized motor plans, of the sort that specify gross movement patterns associated with familiar tool use, not specific kinetic requirements defined according to specific tools and/or the specific environmental constraints for which a given action takes place.

Subjects. Eleven right-handed individuals (seven female; age range, 20-30 years; mean age, 24.7 years) with normal or corrected-to-normal vision participated in the study. None of the participants had any previous history of neurologic or psychiatric illness. All subjects provided informed consent in accordance with the guidelines approved by the University of Western Ontario Health Sciences Research Ethics Board and were naive to the specific goals of the study.

Stimuli and presentation setup. Figure $1 B$ shows the four tools and four handle colors that were used. The complete set included duplicates of each exemplar (tool $\times$ handle color) for a total of 32 items. Tools were presented using the platform and turntable apparatus shown in Figure 1 A. Two Velcro-covered sides were used to attach tools and allowed for the independent presentation of prime and probe events within a trial. On the probe side, a small box was attached to the lower part of the workspace, used by participants to place tools in after their actions within a trial were completed. The platform was specifically adjusted for each individual so that tools and the workspace could be comfortably viewed without the use of mirrors and so that tools could be easily grasped and used with minimal movement of the arm. Specifically, the setup allowed participants to grasp and use tools without the need to lift their elbow from the bed or move their upper arm or shoulder. Grasping without shoulder movement effectively minimizes potential for movementrelated artifacts (Culham, 2004). Tools were presented in the lower right quadrant of the workspace, $\sim 10 \mathrm{~cm}$ to the left of the participant's right hand while in the rest position. Participants were instructed to fixate a small light-emitting diode (LED) that was attached to an adjustable plastic stalk positioned directly above where tools were presented. For both prime and probe events, tools were made visible by brief ( $300 \mathrm{~ms}$ ) illumination of a super-bright white LED attached to a second adjustable stalk. The experiment was otherwise performed in complete darkness.

Tasks. To reduce confusion that would result from intermingling instructions within a run, EXP and CTR tasks were performed in separate runs, each comprised 16 trials. A given trial was made up of the following events: a visual prime event involving $300 \mathrm{~ms}$ illumination of a tool, followed by a $2700 \mathrm{~ms}$ delay with no vision, followed by illumination of a second tool for $300 \mathrm{~ms}$ (Fig. $1 \mathrm{D}$ ). Illumination of the second tool signaled the action (probe) event, in which subjects grasped and used whichever tool was shown and then placed it in the "catch box" before returning to the rest position to wait for the next trial to begin. From the onset of the probe event, there was a $16.5 \mathrm{~s}$ delay period before the next trial began.

On a given trial, prime-probe events involved either the same or different tools with either the same or different colored handles. Both tool identity and handle color were repeated, only tool identity was repeated, only handle color repeated, or both tool identity and handle color were changed (Fig. $1 E$ ). Each of these trial types were shown four times per run, arranged so that specific actions (probe events) were equally represented within a run evenly distributed across trial types. For the EXP task, handle color was irrelevant and participants performed actions according to tool identity, whereas for the CTR task, tool identity was irrelevant and participants performed actions according to handle color (Fig. 1C). For each task, actions were minimal-amplitude movements, involving mostly the wrist, fingers, and thumb, $\sim 3-4 \mathrm{~s}$ in duration. Participants performed a minimum of three EXP runs (with an average of 4.2 runs per subject) and three CTR runs (with an average of 4.1 runs per subject). Eight distinct run orders were created for each EXP and CTR run, and all possible combinations of prime-probe pairings were equally represented across orders. Different run orders were evenly distributed across individuals, randomized for presentation order within individuals, always following an interleaved sequence (e.g., CTR-EXPCTR..., or vice-versa).

Prescan training. Before scanning (maximum of 1 week, minimum of $2 \mathrm{~d}$ ), participants took part in a behavioral training session to learn the arbitrary color-action pairings for the CTR task and to become familiar with the stimuli, trial types, events, and timing. Another important goal of training was to clearly specify and practice the particular actions that were to be performed in the scanner for both EXP and CTR tasks. The 
problems associated with movements of the head while in the scanner were thoroughly explained, and participants understood that their hand actions should not involve movements of the upper arm or shoulder and that their head should be kept still at all times. Minimal-amplitude actions were stressed, emphasizing the use of only the wrist and fingers. Actions were trained to be performed quickly but smoothly, finishing in $\sim 3-4$ s.

Worth noting, for the EXP task, participants were asked to produce an appropriate pantomimed action for each tool (with the tool in hand) without the experimenter specifying particular movement details (e.g., "demonstrate how you might use the spoon to perform a stirring action"). Only subsequently were these actions modified via feedback from the experimenter, shaped according to the various constraints of the MR environment (e.g., space constraints of the bore, minimal-amplitude movements of the wrist and fingers, etc.). In this way, EXP actions were initially generated by participants, presumably at least partly based on internal action representations. This differs from CTR actions whereby specific details of movements were explicitly defined during initial instructions.

After first exposure to tasks and stimuli during training, participants performed 16 trials of each EXP and CTR tasks, presented in separate blocks, balanced for trial types and specific actions within a block. Trial types and timing were the same as used in the actual fMRI experiment, except trials were not spaced so far apart; participants simply signaled the experimenter to initiate new trials once ready. The same turntable apparatus as used in the scanner was used to present tools (Fig. $1 A$ ), and liquid crystal display goggles were used to control participants' vision. These initial 32 trials were performed with visual feedback to allow participants and the experimenter to more easily comment on and adjust the particular mechanics of actions if needed (e.g., to suggest smaller amplitude movements, to emphasize wrist and finger movements, etc.). After these two blocks of trials were performed, two additional blocks of 40 trials per task were performed, this time without visual feedback of actions, as in the fMRI experiment (i.e., tools shown at both prime and probe events were visible for only $300 \mathrm{~ms}$ ). Actions and trial types were equally represented and evenly distributed within blocks, and block order was counterbalanced across individuals. Reaction times were measured as the time taken to release the start button to initiate movements after visual presentation of probe events. For the EXP task, no differences in reaction times were evident when TR (mean \pm SEM, $412 \pm 19.9 \mathrm{~ms}$ ) and TC $(416 \pm 24.5 \mathrm{~ms})$ trials were compared $\left(t_{(10)}=0.20, p=0.85\right)$. Similarly for the CTR task, no differences between CR $(416 \pm 34.7 \mathrm{~ms})$ versus CC $(411 \pm 30.1 \mathrm{~ms})$ trials were found $\left(t_{(10)}=0.13, p=0.90\right)$.

Imaging parameters. Imaging was performed on a 3 Tesla Siemens TIM MAGNETOM Trio MRI scanner. The T1-weighted anatomical image was collected using an ADNI MPRAGE sequence (time to repetition, $2300 \mathrm{~ms}$; time to echo, $2.98 \mathrm{~ms}$; field of view and matrix size, $192 \times 240 \times$ 256; flip angle, $9^{\circ}$; $1 \mathrm{~mm}$ isotropic voxels). Functional MRI volumes were collected using a $\mathrm{T} 2{ }^{*}$-weighted single-shot gradient-echo echo-planar imaging acquisition sequence [time to repetition, $1500 \mathrm{~ms}$; slice thickness, $4 \mathrm{~mm}$; in-plane resolution, $3.3 \times 3.3 \mathrm{~mm}$; time to echo, $30 \mathrm{~ms}$; field of view, $211 \times 211 \mathrm{~mm}$; matrix size, $64 \times 64$; flip angle, $75^{\circ}$; and acceleration factor (integrated parallel acquisition technologies), 2 with generalized autocalibrating partially parallel acquisitions reconstruction]. We used a combination of parallel imaging coils to achieve a good signalto-noise ratio and to enable direct viewing without mirrors or occlusion. We tilted $\left(\sim 30^{\circ}\right)$ the posterior half of the 12-channel receive-only head coil (six channels) and suspended a four-channel receive-only flex coil over the anterior-superior part of the head. Each volume comprised 28 contiguous (no gap) axial-oblique slices, spanning from the most superior point of cortex through ventral fusiform cortex to include approximately two-thirds of cerebellum, providing near whole-brain coverage; volume acquisition space included anterior temporal poles but excluded parts of orbital prefrontal cortex.

Data preprocessing. Imaging data were preprocessed and analyzed using Brain Voyager QX version 2.1.0.1532 (Brain Innovation). Each functional run was assessed for subject head motion by viewing cineloop animations and by examining Brain Voyager motion-detection parame- ter plots after running 3D motion correction algorithms on the untransformed two-dimensional data. No abrupt movements were detected in the animations and no deviations larger than $1 \mathrm{~mm}$ (translations) or $1^{\circ}$ (rotations) per run were observed in motion correction outputs. Motion correction was performed using Brain Voyager QX intrasession alignment options (involving resampling with sinc interpolation) with the reference volume taken as the closest volume to the T1-weighted anatomical scan. Error trials were examined offline from videos recorded using an MR-compatible infrared-sensitive camera that was optimally positioned to record the participant's movements during functional runs (MRC Systems). Because of equipment failure, recordings were not available for 5 of 11 participants. The total number of errors among the six participants with video recordings was 20 (of 864 trials, or 2.3\%). Types of errors included instances in which participants initiated and then inhibited grasping actions at prime events (nine trials, five participants), tools were misoriented or displaced as a result of turning of apparatus (eight trials, four participants), or participants accidently bumped the fixation LED with their hand during grasping (three trials, one participant). Errors were excluded from analysis by assigning these trials predictors of no interest. Notably, errors occurred independent of task and trial type, and there were no instances in which participants performed incorrect actions; tool identity for the EXP task and handle color for the CTR task were correctly distinguished, and corresponding actions were executed.

Functional data were preprocessed with linear trend removal and underwent high-pass temporal frequency filtering to remove frequencies below three cycles per run and aligned to anatomical volumes, which were then transformed into standard stereotaxic space (Talairach and Tournoux, 1988). Data were spatially smoothed for group analysis using a Gaussian kernel of $6 \mathrm{~mm}$ (full-width at half-maximum).

Data analyses. By definition, EXP and CTR tasks differed with respect to which stimulus dimension was relevant for action planning; actions were defined by tool identity for the EXP task, whereas actions were defined by handle color for the CTR task. As such, for main analysis, trial type congruency and RS were defined differently according to task. For the EXP task, congruent trials involved repeated tool identity (TR) and incongruent trials involved changed tool identity (TC), and RS was predicted to correspond with TC $>$ TR activity (EXP-RS). For the CTR task, congruent trials involved repeated color (CR) and incongruent trials involved changed color (CC), and RS was predicted to correspond with CC $>$ CR activity (CTR-RS). In other words, we simplified the design for our main analysis by defining trial types for the EXP task independent of handle color and trial types for the CTR task independent of tool identity.

Although RS as a result of repeated versus changed task-irrelevant stimulus dimensions (handle color for the EXP task and tool identity for the CTR task) was theoretically possible, we consider this unlikely. First, short (300 ms) exposure to stimuli placed high demands on processing of task-relevant stimulus dimensions; time to process task-irrelevant stimulus dimensions was limited. Second, results of our previous behavioral study involving a similar paradigm revealed that, when distinct action tasks are blocked, as in the current study (i.e., presented in separate runs), task-irrelevant stimulus dimensions are ignored and do not lead to behavioral priming (Valyear et al., 2011). Evidently, these assumptions were verified by subsequent analyses (in addition to the main analysis) showing no effects of repetition of task-irrelevant stimulus dimensions for either task, according to direct contrasts performed at the wholebrain voxelwise level and according to analysis of percentage BOLD signal change values (\%-BSC) extracted from areas identified in the main analysis.

All analyses were based on a group-level random-effects general linear model (GLM) including all EXP and CTR runs with five predictors specified: TC, TR, CC, CR, and an error predictor. Each predictor was modeled as a four-volume ( $6 \mathrm{~s})$ boxcar function aligned to the start of prime events convolved with Brain Voyager QX default two-gamma function designed to estimate spatiotemporal characteristics of the BOLD response. "Dummy" predictors (columns of all zeros in the design matrix) were included when corresponding trial types were not present within a run (i.e., CC and CR for EXP runs, TC and TR for CTR runs, and error 


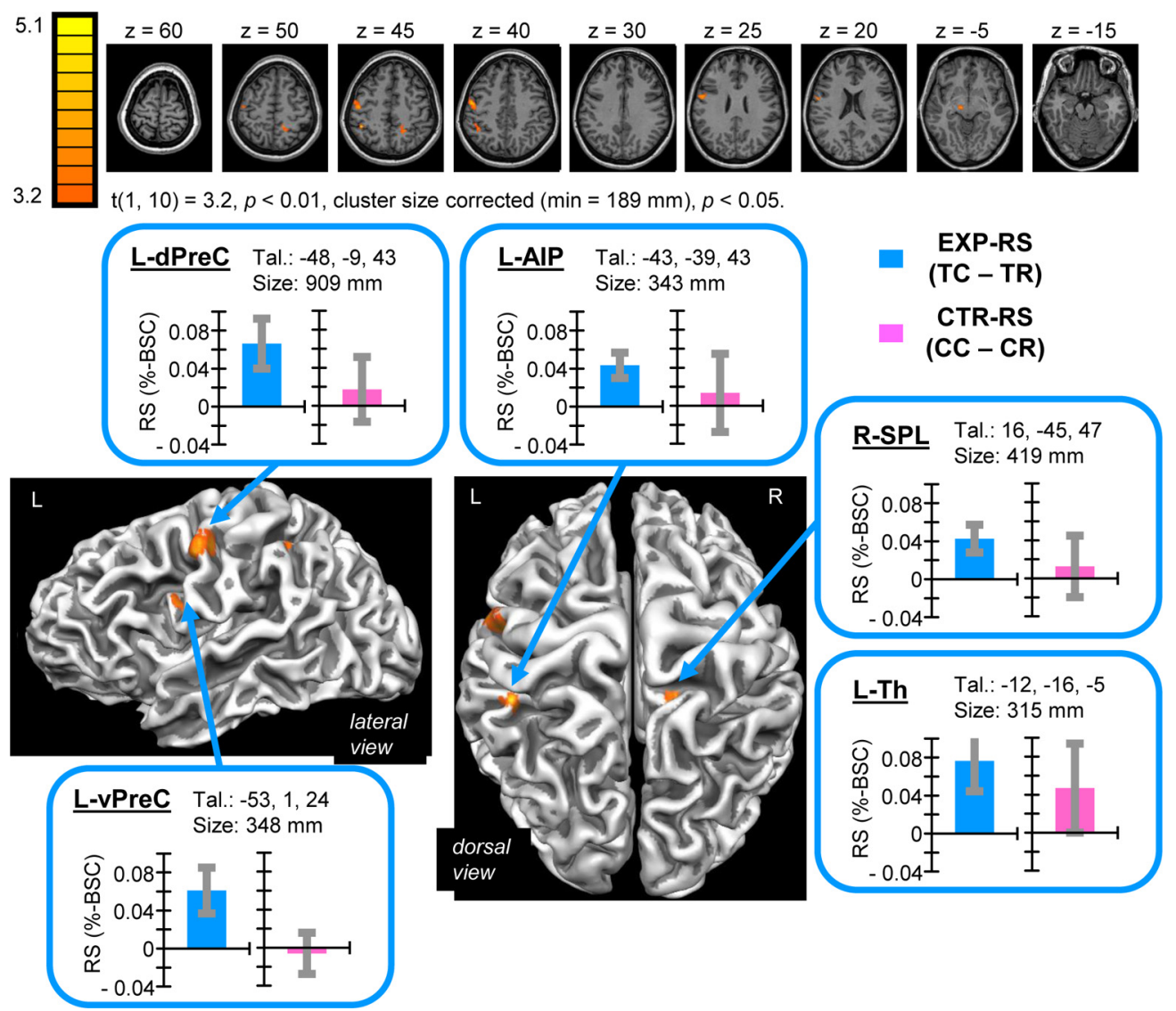

Figure 2. Results of single-contrast analysis showing RS for tool-use demonstration. Activations reflect areas identified by the contrast TC $>$ TR indicative of EXP-RS. Group results are superimposed on two-dimensional axial slices (top) as well as three-dimensional cortical surface reconstructions (bottom) of a single subjects' anatomy. Areas showing significant EXP-RS included L-AIP, L-dPreC, L-vPreC, and L-Th, as well as R-SPL. Mean center-of-mass Talairach coordinates and volume size of areas are indicated. Group mean difference scores corresponding to predictions regarding RS for EXP and CTR tasks, respectively, indicate specificity of EXP-RS, although CTR-RS approached significance levels for L-Th exclusively. Error bars reflect $95 \%$ confidence intervals based on SEs of the corresponding mean difference scores across individuals.

predictors for runs without errors). Each run was percent-transformed before GLM analysis.

First-level analysis included two independent contrasts used to identify voxels active for either task versus rest, EXP (TC and TR) $>$ rest and CTR $(C C$ and CR $)>$ rest. Resultant activation maps were set to a statistical threshold of $t=3.2$ ( $p<0.01$, uncorrected for multiple comparisons), corrected for multiple comparisons using Brain Voyager QX cluster-level statistical threshold estimator, found to indicate a minimum cluster size of $189 \mathrm{~mm}^{3}$ ( $\left.p<0.05\right)$. An inclusion mask comprising all voxels activated by either contrast was used to constrain all subsequent analyses.

Main analyses included two distinct approaches, single-contrast analysis and factorial analysis. Single-contrast analysis included two independent contrasts, TC $>$ TR used to identify areas showing RS for the EXP task, and CC $>$ CR used to identify areas showing RS for the CTR task. Resultant activation maps were set to a statistical threshold of $t=3.2$ [ $p<0.01$, cluster size corrected (minimum of $189 \mathrm{~mm}^{3}$ ), $\left.p<0.05\right]$. Factorial analysis included task (two levels, EXP and CTR) and trial type congruency (two levels, congruent and incongruent) as factors, performed using Brain Voyager QX ANOVA/ANCOVA module. Congruency was defined in the same way as with single-contrast analysis: TR and CR were defined as congruent, whereas TC and CC were defined as incongruent. This analysis allowed for generation of statistical activation maps at the whole-brain voxelwise level according to significant main effects of task, congruency, and the interaction term. Resultant activation maps were set to a statistical threshold of $F=10.2[p<0.01$, cluster size corrected (minimum of $189 \mathrm{~mm}^{3}$ ), $p<0.05$ ].

\section{Results}

First-level analysis identified voxels active for either task versus rest, used to define an inclusion mask to constrain all subsequent analyses (for details, see Materials and Methods). As expected, both tasks led to widespread activation of sensorimotor areas, including primary motor and somatosensory, dorsal and ventral premotor, posterior parietal and cingulate motor areas, as well as the thalamus, basal ganglia, and cerebellum. Parietofrontal activations were observed bilaterally although with clear left hemisphere prevalence, consistent with the fact that participants performed actions with their right hand. Bilateral medial occipital cortex including primary visual areas, as well as lateral occipitotemporal cortex (primarily in left hemisphere), were also activated for both tasks.

Single-contrast analysis: RS of tool use pantomimed actions RS for the EXP task (EXP-RS) was predicted to correspond with TC $>$ TR activity within areas important for tool use planning and execution. The contrast TC $>$ TR identified five distinct areas of activity at reliable statistical thresholds: left-lateralized anterior intraparietal (L-AIP), ventral precentral (L-vPreC), dorsal precentral (L-dPreC), and thalamus (L-Th), as well as right hemisphere superior parietal lobule (R-SPL) (Fig. 2). No areas were found to show the reverse pattern of activity (i.e. TR $<\mathrm{TC}$ ). Post hoc analysis of trial-type-specific \%-BSC values extracted 
from identified areas revealed that RS was specific to the EXP task and familiar tool use actions and did not generalize to the CTR task and arbitrary color-defined actions, although CTR-RS effects approached significance $(p=0.073)$ for L-Th exclusively. Note that, for the EXP task, these results were already determined by the contrast used to define areas, shown merely for descriptive purposes, whereas for the CTR task, data are independent from the contrast used to define areas and thus comparisons of CC-CR activity based on \%-BSC values provide an unbiased test of CTR-RS in these areas (as prescribed according to Kriegeskorte et al., 2009). The results indicate specificity of RS for familiar tool-action associations and not novel arbitrary color-action associations within these areas.

EXP-RS is interpreted as arising from the activation of toolspecific motor programs relevant for conventional use in response to prime events involving passive viewing of tools. Reactivation of shared motor programs after repeated tools for TR trials results in decreased BOLD activity relative to TC trials, which instead involve distinct tool-specific motor activity for prime and probe events. This interpretation is conceptually equivalent to the idea that prime events act as cues to subsequent actions, prompting activation of associated motor programs. Below (see Discussion), we consider the possibility that EXP-RS can be understood with respect to neural mechanisms previously identified with monkey electrophysiology in the context of object grasping.

Good correspondence with previous tool-related neuroimaging studies as well as relevant neuropsychological findings supports the hypothesis that areas showing EXP-RS correspond with areas important for planning and execution of familiar tool use. The locations of areas showing EXP-RS correspond well with the locations of areas identified with previous neuroimaging studies involving familiar tool use pantomimed actions (Johnson-Frey, 2004; Lewis, 2006). Strongly left-lateralized inferior parietal and inferior and middle frontal activity have been consistently identified independent of the hand used to perform tool use actions (Johnson-Frey et al., 2005; Bohlhalter et al., 2009; Króliczak and Frey, 2009), closely overlapping with the locations of L-AIP, L-vPreC, and L-dPreC areas showing EXP-RS in the current study (see also Rumiati et al., 2004; Fridman et al., 2006). Furthermore, neuropsychological evidence has indicated that damage to these same areas often results in ideomotor apraxia (IM), a disorder characterized by selective impairments in performing learned skilled actions not explained by elementary motor or sensory deficits or related to general problems with cognition, comprehension, or attention (Rothi and Heilman, 1997). Modern lesion analysis methods have consistently indicated that leftlateralized inferior parietal cortex, including supramarginal gyrus and surrounding intraparietal cortex, as well as inferior/ middle frontal gyri and surrounding precentral cortex, are sites of maximal lesion overlap in patients with IM and impaired performance of tool use pantomime (Haaland et al., 2000; Buxbaum et al., 2005; Goldenberg and Spatt, 2009). In summary, areas showing EXP-RS in the current study overlap closely with areas previously hypothesized to underlie the motoric aspects of skilled actions with tools.

Notably, the locations of areas showing EXP-RS also correspond well with the locations of areas identified in previous fMRI RS studies involving real actions (Dinstein et al., 2007; Króliczak et al., 2008; Chouinard and Goodale, 2009; Hamilton and Grafton, 2009), as well as areas recently found to show predictive coding of upcoming grasp and reach actions according to distinct spatial (voxelwise) activation patterns (Gallivan et al., 2011).
Also, EXP-RS in L-AIP appears close in location to activity reported in previous studies involving real grasping; center-ofmass Talariach coordinates for L-AIP were found to be $1 \mathrm{~mm}$ lateral to $95 \%$ confidence intervals according to the mean coordinates of grasp-defined AIP activity based on nine published studies (Binkofski et al., 1998; Culham et al., 2003; Frey et al., 2005; Begliomini et al., 2007a,b; Cavina-Pratesi et al., 2007, 2010; Króliczak et al., 2007, 2008): $x=-36$ to $-42, y=-35$ to -43 , $z=40$ to 48 . L-vPreC showing EXP-RS is close in location to what others have called ventral premotor cortex for grasping (Cavina-Pratesi et al., 2010) and tool use (Fridman et al., 2006), whereas L-dPreC showing EXP-RS is lateral to dorsal premotor cortex as typically defined for grasping (Króliczak et al., 2008).

We find it surprising that EXP-RS was not also detected within occipitotemporal areas part of the ventral visual pathway specialized for object recognition. Familiar tool use has been hypothesized to involve cooperation between ventral stream and inferior parietal areas, whereby ventral stream inputs are thought to provide information about tool identity and function important for specification of motor programs underlying their conventional use (Milner and Goodale, 1995, pp 202-204; Valyear and Culham, 2010). The current findings appear at odds with this hypothesis and may be interpreted as support for a nonconceptual route to learned actions (Riddoch et al., 1988; Pilgrim and Humphreys, 1991; Rumiati and Humphreys, 1998). Several previous neuropsychological findings have shown that reasonably proficient tool use can persist despite profound loss of conceptual object knowledge as a result of bilateral damage to anterolateral temporal cortex (Sirigu et al., 1991; Buxbaum et al., 1997; LauroGrotto et al., 1997). Notably, however, Hodges et al. (1999, 2000) have instead stressed the importance of spared conceptual object knowledge and anterolateral temporal areas in enabling correct performance of conventional tool use and suggest several compensatory factors that can account for the occasional sparing of seemingly normal tool use ability in the face of conceptual knowledge impairments. Other findings also implicate ventral stream areas as important for tool use (Carey et al., 1996; Creem and Proffitt, 2001; Mahon et al., 2007).

In light of these previous findings, we offer the following possible explanations to account for the absence of EXP-RS within the ventral stream. First, theoretical models of ventral stream involvement in tool use specify a role for these areas in sending forward information about object identity to inferior parietal (and frontal premotor) areas. Previous findings have indicated that BOLD activity is more strongly correlated with local field potentials, which reflect the inputs to and local processing within an area, versus single and multiunit spiking activity, which reflect long-range outputs (Logothetis et al., 2001; Logothetis and Wandell, 2004). Thus, it is possible that activation changes may be detected at the level of inputs to an area (inferior parietal cortex) but missed at the output level (ventral stream areas), which may in fact be more problematic for fMRI RS designs (Tolias et al., 2001). Second, if we consider the role of the ventral stream in tool use as specifically important for the identification of tools, processing demands within the ventral stream may have been minimized in the current study as a result of the use of a relatively small set of distinct tools for which corresponding actions were practiced before scanning. These factors may have reduced demands for processing tool identity, dampening activity and in turn the potential to detect EXP-RS within ventral stream areas. Similarly, short tool presentation durations (300 ms) may have minimized the overall strength of activity in ventral stream areas and thus the likelihood of observing EXP-RS. The above ac- 


\section{1}

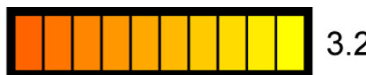

$\mathrm{t}(1,10)=3.2, p<0.01$, cluster size

corrected $(\min =189 \mathrm{~mm}), \mathrm{p}<0.05$

L-alNS

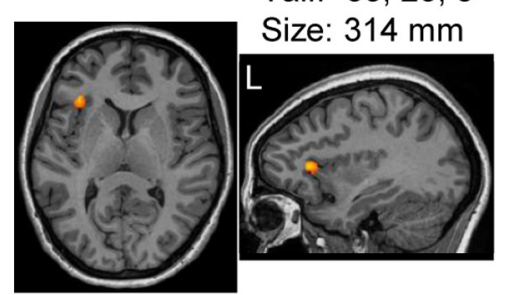

B-Calc

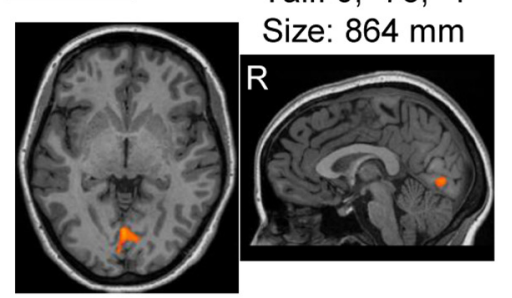

$\underline{\mathrm{R}-\mathrm{pCLS}}$

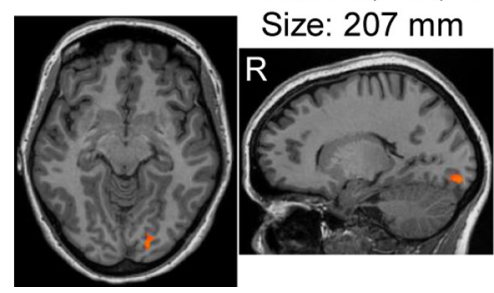

Tal.: $26,-82,-7$

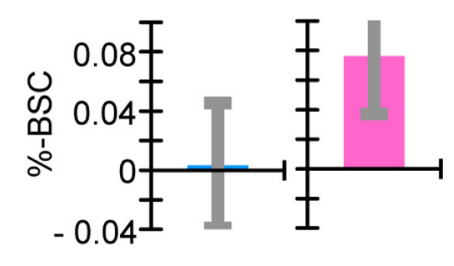

Figure 3. Results of single-contrast analysis showing RS for color-defined CTR actions. Activations reflect areas identified by the contrast CC $>$ CR indicative of CTR-RS. Areas showing significant CTR-RS included L-alNS, B-Calc, and R-pCLS. Each group-defined area is shown on two-dimensional axial and sagittal slices of a single subjects' anatomy shown with respect to the mean centerof-mass Talairach coordinates of the area. Area volume sizes are also indicated. Group mean difference scores corresponding to predictions regarding RS for EXP and CTR tasks, respectively, indicate specificity of RS for the CTR task. Error bars reflect 95\% confidence intervals based on SEs of the corresponding mean difference scores across individuals.

counts are admittedly speculative and require verification. Clearly, future investigations are needed to clarify the potential importance of ventral stream areas in planning and execution of familiar tool use.

\section{Single-contrast analysis: RS of color-defined control actions} To explore the possibility that distinct brain areas may show RS specific to the CTR task, we performed the contrast CC $>$ CR. The results revealed three distinct areas of activity at reliable statistical thresholds: left anterior insula (L-aINS), bilateral calcarine (B-Calc), and right posterior collateral sulcus (R-pCLS) (Fig. 3). No areas were found to show the reverse pattern of activity (i.e., $\mathrm{CR}<\mathrm{CC}$ ). Analysis of trial-type-specific \%-BSC values extracted from identified areas revealed that RS was specific to the CTR task and did not generalize to the EXP task. These findings suggest a selective role for these areas in mediating color-defined CTR actions.

These results were not predicted a priori. Nonetheless, we suggest that CTR-RS reflects the recruitment of distinct cognitive strategies used to perform the CTR task. Mohr et al. (2006) reported an area close in location to the L-aINS area we have identified (they referred to as left ventral prefrontal cortex) that was selectively active for encoding and maintaining color (vs spatial) information over a delay period. More recently, Yee et al. (2010) found greater delay-period activity for color versus shape information in what appeared to be the same left prefrontal area, and the strength of activity in this area was related to better performance on the color task. Notably, both of these previous studies also reported concurrent color-specific activations within posterior medial occipital areas, in close proximity to areas B-Calc and R-pCLS showing CTR-RS in the current study. Mohr et al. (2006, p 4468) proposed that left ventral prefrontal cortex "might control the rehearsal of color information through a cross talk with more posterior visual areas." The activation we observed in the current study showing CTR-RS may be interpreted similarly. To plan and perform CTR actions accordingly, L-aINS may have maintained color-defined rules in mind and coordinated with early visual areas B-Calc and R-pCLS for which activity may be more directly attributable to distinguishing handle colors. Evidently, handle color of tools shown as prime events must have been processed by this network such that when color was repeated for CR trials, BOLD activity was relatively reduced. An important implication of this interpretation is that RS can be used to distinguish brain areas distinctly involved in planning and execution of different types of actions according to distinct underlying cognitive strategies.

\section{Factorial analysis}

Complimentary to single-contrast analyses, we performed whole-brain voxelwise factorial analysis, including task (EXP, CTR) and trial-type congruency (congruent, incongruent) as independent factors.

Results of the interaction term identified a single area of activation localized to L-vPreC, centered within precentral sulcus and extending anteriorly to include posterior aspects of inferior frontal gyrus (pars opercularis) (Fig. 4). Post hoc analysis of trialtype-specific \%-BSC values revealed that the interaction was driven by RS for the EXP task (i.e., TC $>$ TR) exclusively (Table 1). A nonsignificant trend in the opposite direction predicted for CTR-RS was evident (i.e., CR > CC). These results demonstrate that, of the areas showing EXP-RS identified in the singlecontrast analysis (Fig. 2), only L-vPreC showed specificity for EXP-RS versus CTR-RS that was robust enough to be identified with voxelwise factorial analysis. Consistently, $40 \%$ of voxels ( 82 of 203) comprising this area overlapped with voxels comprising the L-vPreC area identified with single-contrast analysis. Evidently, nonsignificant trends in the direction of CTR-RS observed in the four other areas showing EXP-RS identified with single-contrast analysis (Fig. 2) prevented detection of these areas according to a significant task $\times$ congruency interaction specified by factorial analysis. Consequently, strictly speaking, interpretation of specificity of RS for the EXP task and not the CTR task for 


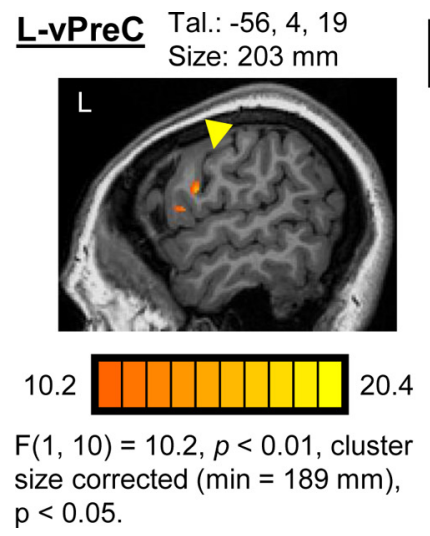

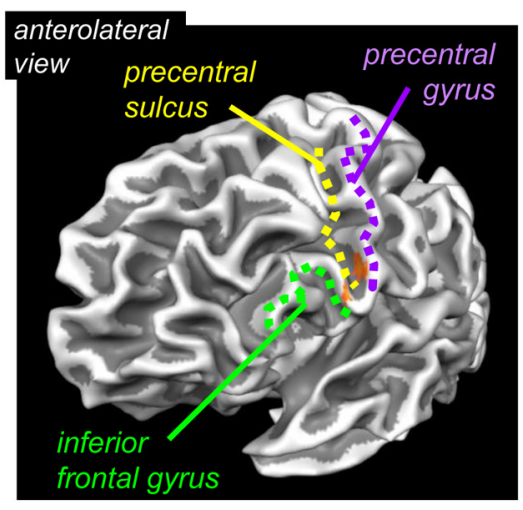

EXP-RS

(TC - TR)

CTR-RS

$(C C-C R)$

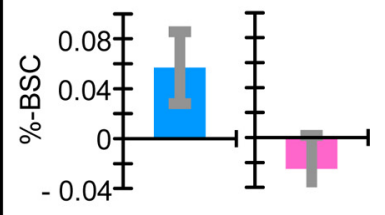

inferior

frontal gyrus

Figure 4. Results of factorial analysis showing task $\times$ congruency interaction. A single area of activation in L-vPreC showed a significant task $\times$ congruency interaction based on group voxelwise factorial analysis. Group results are shown on a single sagittal slice and three-dimensional cortical surface reconstruction of a single subjects' anatomy. Activity was localized to the inferior precentral sulcus and posterior inferior frontal gyrus (pars opercularis). Group mean difference scores corresponding to predictions regarding RS for EXP and (TR tasks indicate directionality of effects. Error bars for RS measures according to task reflect $95 \%$ confidence intervals based on SEs of the corresponding mean difference scores across individuals. The interaction is driven by significant RS for the EXP task (TC > TR) but not the CTR task, which instead shows a nonsignificant trend in the opposite direction predicted to correspond with RS (CR > CC). Results reveal that RS in L-vPreC is specific to actions involving familiar tool use pantomimes.

Table 1. Results of factorial analysis

\begin{tabular}{|c|c|c|c|c|c|c|c|c|c|c|}
\hline \multirow[b]{3}{*}{ Brain regions } & \multicolumn{3}{|c|}{ Talairach coordinates } & \multirow{3}{*}{$\begin{array}{l}\text { Volume } \\
\left(\mathrm{mm}^{3}\right)\end{array}$} & \multirow{2}{*}{\multicolumn{3}{|c|}{ RM-ANOVA, $F_{(1,10)}$}} & \multirow{2}{*}{\multicolumn{3}{|c|}{ Pairwise $t$ test, $t_{(1,10)}$}} \\
\hline & \multicolumn{3}{|c|}{ Mean center-of-mass } & & & & & & & \\
\hline & $x$ & $y$ & $z$ & & ME of Task & ME of congruency & Interaction & EXP versus CTR & EXP-RS & CTR-RS \\
\hline L-SMG & -55 & -27 & 35 & 1595 & $p<0.001^{a}$ & $p=0.46$ & $p=0.057^{*}$ & $\operatorname{EXP}>$ CTR, $p<0.001^{b}$ & $\mathrm{TC}>\mathrm{TR}, p=0.038^{*}$ & $\mathrm{CR}>\mathrm{CC}, p=0.64$ \\
\hline R-SMG & 57 & -31 & 32 & 452 & $p<0.005^{a}$ & $p=0.43$ & $p=0.51$ & $\operatorname{EXP}>$ CTR, $p<0.005^{b}$ & $\mathrm{TC}>\mathrm{TR}, p=0.76$ & $\mathrm{CR}>\mathrm{CC}, p=0.37$ \\
\hline L-BG & -23 & -4 & 7 & 205 & $p<0.005^{a}$ & $p<0.05^{b}$ & $p=0.63$ & $\mathrm{EXP}>\mathrm{CTR}, p<0.005^{b}$ & $\mathrm{TC}>\mathrm{TR}, p=0.12$ & $C \mathrm{C}>\mathrm{CR}, p=0.13$ \\
\hline L-alNS & -30 & 21 & 7 & 1040 & $p<0.005^{a}$ & $p=0.23$ & $p=0.11$ & CTR $>$ EXP, $p<0.005^{b}$ & $\mathrm{TR}>\mathrm{TC}, p=0.80$ & $C C>C R, p<0.01^{b}$ \\
\hline \multicolumn{11}{|c|}{$\begin{array}{l}\text { Regions defined by a main effect of } \\
\text { congruency }\end{array}$} \\
\hline L-dPreC & -48 & -7 & 42 & 285 & $p=0.21$ & $p<0.005^{a}$ & $p=0.10$ & CTR $>$ EXP, $p=0.21$ & $\mathrm{TC}>\mathrm{TR}, p<0.001^{b}$ & $C C>C R, p=0.19$ \\
\hline L-Calc & -3 & -73 & 1 & 248 & $p=0.11$ & $p=0.005^{a}$ & $p=0.071^{*}$ & CTR $>$ EXP, $p=0.11$ & $\mathrm{TC}>\mathrm{TR}, p=0.55$ & $\mathrm{CC}>\mathrm{CR}, p<0.005^{b}$ \\
\hline L-Th & -10 & -16 & -4 & 599 & $p=0.10$ & $p<0.005^{a}$ & $p=0.52$ & $\mathrm{EXP}>\mathrm{CTR}, p=0.10$ & $\mathrm{TC}>\mathrm{TR}, p<0.005^{b}$ & $C \mathrm{C}>\mathrm{CR}, p<0.01^{b}$ \\
\hline
\end{tabular}

these areas should be made more cautiously, whereas EXP-RS specificity for L-vPreC was statistically robust and warrants unconstrained interpretation.

As a secondary interest, we wanted to test for areas showing preferential activity for either EXP or CTR tasks independent of RS. These additional analyses were important given that conventional contrast methods may reveal task (or stimulus) specificity not detected by RS methods and vice versa (Grill-Spector and Malach, 2001). Whereas EXP-RS was hypothesized to specifically target areas underlying the transformation of visually defined tool properties to corresponding motor programs for their conventional use, preferential activity for the EXP versus CTR task may reveal areas that mediate other types of processes important for tool use.

These analyses also provided the opportunity to evaluate EXP and CTR tasks with respect to potential differences in motor and/or somatosensory aspects. An important strength of the RS method as defined in the current study is that key comparisons (i.e., $\mathrm{TC}>\mathrm{TR}$ and $\mathrm{CC}>\mathrm{CR}$ ) involve trials that include the same executed actions, so that resultant activations are not attributable to potential motor or somatosensory confounds (e.g., differences in extent, complexity, or duration of movements or haptic feedback). Conversely, direct comparison of EXP versus CTR tasks may reveal activations that reflect such potential confounding factors, predicted to correspond with preferential activity in primary motor and/or somatosensory areas. It was important to evaluate this possibility given that task-specific RS may in fact interact with such differences in overall task demands. That is, it is possible that greater sensorimotor demands associated with the EXP versus CTR task may have contributed to the specificity of EXP-RS as detected in parietofrontal areas.

Results of factorial analysis of main effects of task revealed six distinct areas of activity at reliable statistical thresholds: left (L-) and right (R-) supramarginal gyrus (SMG), left basal ganglia (LBG), L-aINS, left posterior intraparietal sulcus (L-pIPS), and 


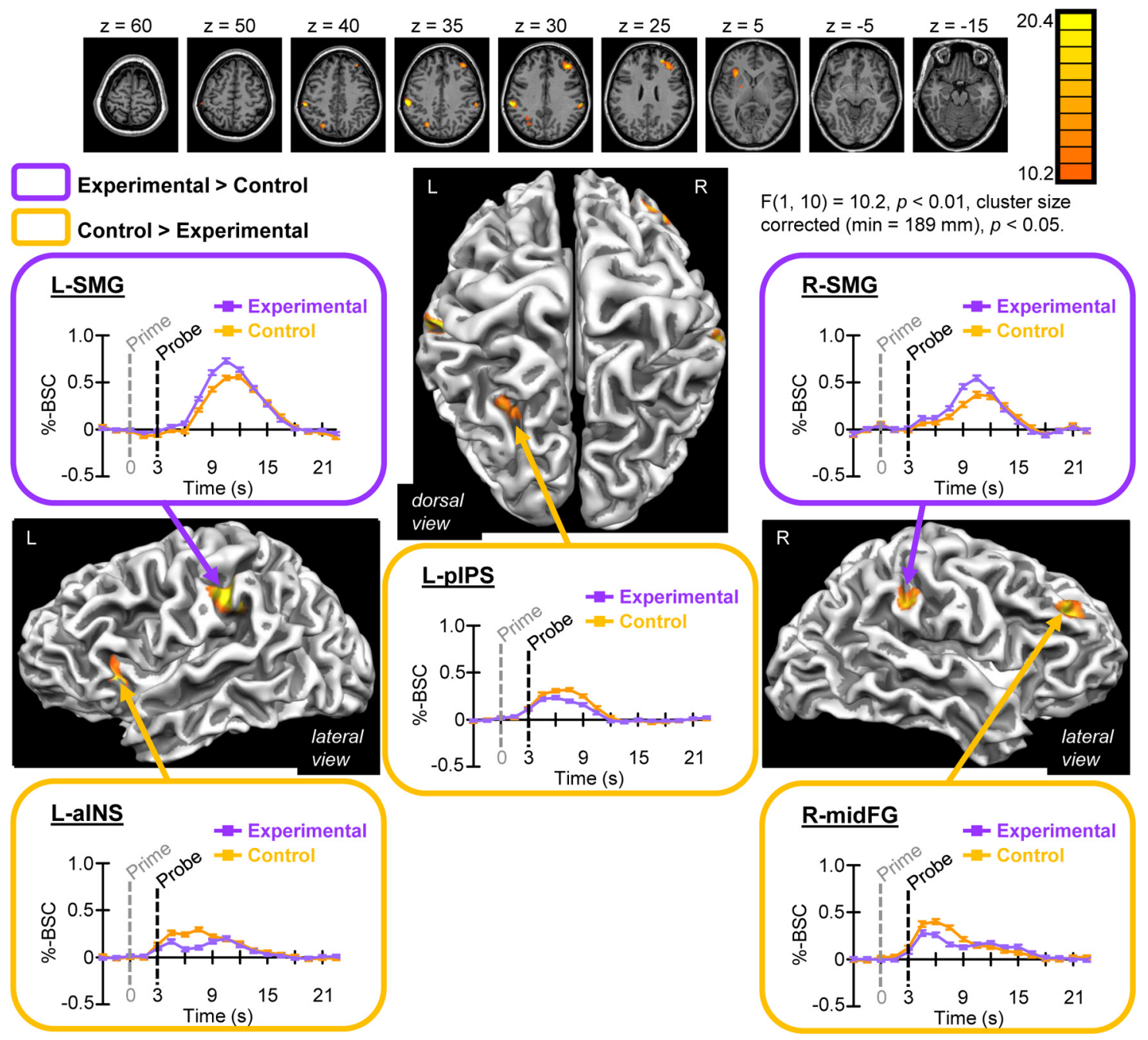

Figure 5. Results of factorial analysis showing main effects of task. Activations reflect areas showing a significant main effect of task based on group voxelwise factorial analysis. Group results are shown on two-dimensional axial slices (top) as well as three-dimensional cortical surface reconstructions of a single subjects' anatomy. Event-related averaged \%-BSC values extracted from each area shown as a function of time per task indicate directionality of effects. Error bars reflect SEMs. The EXP task preferentially activated SMG bilaterally (with left hemisphere prevalence), as well as L-BG (visible in slice $z=-5$; for details, see Table 1), whereas the CTR task preferentially activated L-aINS, L-pIPS, and R-midFG. See Table 1 for volume size of areas, center-of-mass Talairach coordinates, and results of follow-up statistical tests.

right middle frontal gyrus (R-midFG) (Fig. 5). Post hoc analysis of task-specific \%-BSC values revealed directionality of effects ( $\mathrm{Ta}$ ble 1), also evident visually as task-specific \%-BSC time courses extracted from areas shown in Figure 5. The EXP task preferentially activated L-SMG, R-SMG, and L-BG, whereas the CTR task preferentially activated L-aINS, L-pIPS, and R-midFG. These results do not support the possibility that EXP and CTR tasks differed in sensorimotor demands.

Post hoc analysis of \%-BSC values extracted from areas showing preferential activity for the EXP task revealed close to significant EXP-RS for L-SMG exclusively (TC $>$ TR, $t=2.39, p=$ 0.038 ; note that, after Bonferroni correction for multiple comparisons, significance was set at $p<0.02$; see Table 1 ). L-SMG was situated anterior (extending ventrally) to the L-AIP area detected in the single-contrast analysis showing EXP-RS (Fig. 2). In other words, sensitivity to EXP-RS within left inferior parietal cortex was found to be maximal for anterior aspects of intraparietal cortex (L-AIP area) and was reduced to nonsignificant levels for more ventral anterior aspects of supramarginal gyrus (L-SMG area). EXP-RS is dependent on sensitivity to visually defined aspects of tools, and, although speculative, this posterior-to- anterior superior-to-inferior continuum of sensitivity may relate to findings from previous neuroimaging studies of grasping that have identified distinct posterior-to-anterior visual-to-motor sensitivity gradients within intraparietal cortex (Culham, 2004; Stark and Zohary, 2008; Gallivan et al., 2011). Notably, the location of L-SMG has been consistently reported in previous studies involving real tool use pantomimed actions (Rumiati et al., 2004; Johnson-Frey et al., 2005; Fridman et al., 2006; Króliczak and Frey, 2009). That areas showing preferential activity for the EXP task were non-overlapping with areas showing EXP-RS demonstrates that task specificity can be differentially revealed with conventional contrast versus RS methods. In summary, we consider bilateral SMG and L-BG revealed as preferentially active for the EXP task as important for familiar tool use, perhaps more closely related to motor/somatosensory versus visual aspects of action planning and execution.

Conversely, L-aINS, L-pIPS, and R-midFG were revealed as preferentially active for the CTR versus EXP task. Analysis of $\%$-BSC values from these areas showed significant CTR-RS for L-aINS (Table 1), and, consistently, 15\% (156 of 1040) of voxels for this area overlapped with voxels comprising the L-aINS area 
showing CTR-RS identified with single-contrast analysis (Fig. 3). Thus, L-aINS showed specificity for both the CTR task in general and CTR-RS, taken as additional support for our interpretation of this area as involved in the maintenance of arbitrary coloraction associations specific to the CTR task. A nonsignificant trend toward sensitivity to CTR-RS was evident in R-midFG, whereas L-pIPS showed no evidence of RS for either task.

Finally, main effects of congruency identified activity in L-dPreC, L-Th, and left calcarine (L-Calc) (Table 1). Analysis of $\%$-BSC values from L-dPreC indicates that these effects were driven by EXP-RS, consistent with the fact that $57 \%$ of voxels (148 of 285) from this area overlapped with $\mathrm{L}-\mathrm{dPreC}$ showing EXP-RS identified with single-contrast analysis (Fig. 2). Conversely, L-Calc was driven by significant CTR-RS and showed $85 \%$ voxel overlap (201 of 248 ) with B-Calc identified with single-contrast analysis (Fig. 3). Last, L-Th showed a genuine main effect of congruency, sensitive to both EXP-RS and CTR$\mathrm{RS}$, which suggests a generalized role for this area in planning and execution of actions for both tasks, sensitive to action-relevant stimulus properties independent of information type, familiarity of associations, and putative task differences in underlying cognitive strategies.

\section{Discussion}

The current study identifies areas important for planning and execution of tool use pantomimed actions and suggests potential underlying neural response mechanisms. EXP-RS is thought to reflect sensitivity to passively viewed action-relevant tool properties in areas devoted to transformation of this information to corresponding motor programs. These results are hypothesized to relate to neural processes fundamental to action planning, selection, and execution known as affordance specification whereby action-relevant object properties (affordances) are visually represented in sensorimotor areas (Cisek and Kalaska, 2010). The current study extends previous findings based on physical object properties to reveal affordance activity driven by learned properties of tools related to their common use. Specificity for EXP-RS versus CTR-RS is thought to reflect differences in the extent of previous sensorimotor experience and thus the strengths of associations underlying affordance-driven activity. Consistently, the temporary nature of color-action associations performed in the CTR task is considered to account for the recruitment of distinct brain areas thought to involve distinct cognitive-motor strategies.

\section{Affordance specification for tools}

The neural response mechanisms underlying EXP-RS identified in the current study may correspond with response characteristics of neurons identified in monkey sensorimotor areas in the context of object grasping and manipulation. Neurons in monkey AIP and ventral premotor (F5) areas show motor selectivity for particular types of grasps thought to reflect specification of corresponding motor parameters (Jeannerod et al., 1995). A subset of AIP/F5 neurons (canonical neurons) show visual response selectivity for object properties (size, shape, orientation) during passive fixation in agreement with motor selectivity during grasping (Raos et al., 2006; Baumann et al., 2009). These response characteristics have been hypothesized to mediate transformation of visual object properties to corresponding motor programs for grasping (Fagg and Arbib, 1998). Activation during object fixation is thought to reflect a process of affordance specification whereby the properties of objects relevant for action are visually represented within the same neurons responsible for the spec- ification of corresponding motor parameters underlying those actions. These same mechanisms underlying affordance specification are evident in other sensorimotor control areas, including dorsal premotor F2 (Raos et al., 2004) and primary motor cortex M1 (Umiltà et al., 2007), and are considered fundamental to action planning, selection, and execution (Cisek and Kalaska, 2010).

We hypothesize that similar mechanisms exist in humans and that, for highly familiar tools, affordance specification includes activation of motor programs related to their common use. The current findings can be understood as arising from the activation of distinct populations of neurons tuned to different tools according to differences in the motoric aspects of the actions for which they are associated. Repeated presentation of tools for TR trials results in activation of more of the same neural populations (and reduced BOLD signal) relative to TC trials involving distinct tools that activate relatively distinct neural populations. This interpretation is supported by the fact that areas showing EXP-RS correspond well with areas thought to underlie motoric aspects of tool use according to both previous neuroimaging and neuropsychological findings (see Results).

Additional support for this hypothesis stems from previous fMRI RS studies involving real actions. These studies have shown good congruency between response properties of sensorimotor areas inferred from fMRI RS and those derived from monkey neurophysiology. Repeated versus non-repeated grasping (Króliczak et al., 2008) and intransitive (i.e., non-object-related) hand gestures (Dinstein et al., 2007; Chouinard and Goodale, 2009; Hamilton and Grafton, 2009) revealed RS in a number of putative sensorimotor control areas, including AIP, consistent with action-specific coding as established with neural recording methods in monkey area AIP. Furthermore, Króliczak et al. (2008) showed RS for object shape independent of grasp orientation in AIP, which indicates response sensitivity to visually defined aspects of stimuli (see also Chouinard and Goodale, 2009), consistent with canonical-neuron-like responses (see also Grèzes et al., 2003). Finally, mirror neurons are interspersed with canonical neurons in monkey areas AIP and F5 and show motor selectivity for executed and (often matched) visual selectivity for observed actions (Rizzolatti and Craighero, 2004). Consistent with these response properties, Dinstein et al. (2007) showed RS for both repeated executed and observed actions in AIP and ventral (and dorsal) premotor cortex. Activations consistently reported in these studies correspond well with the locations of areas showing EXP-RS in the current study. These findings strengthen interpretation of the current results as mediated by similar neural response properties as those identified in monkey sensorimotor areas underlying visual specification of object affordances. The current findings make important new advancements in this area by providing evidence that affordance-driven activity in humans in response to viewing tools includes specification of learned action-relevant properties related to their conventional use.

A number of recent behavioral findings have provided support for a similar account of the visual specification of tool affordances. Viewing tools can modulate reaction times to initiate subsequent actions known as behavioral priming. Critically, these effects have been shown to reflect access to information about predicted tool-specific action outcomes according to previous knowledge of their common use (Masson et al., 2011). These findings coincide nicely with our interpretation of the current results. Notably, EXP-RS was detected in the current study despite the absence of behavioral priming (i.e., reaction times for TR and TC trials showed no differences during prescan training). 
However, no evidence of behavioral priming can be viewed as a strength given that TC $>$ TR activity is thus not attributable to mere differences in processing times. Follow-up studies may directly compare fMRI RS and behavioral priming of tool use. Unraveling their potential correspondence is an exciting direction for future research.

Specification of affordances is not only considered important for performing actions but also for selecting between potential alternatives. Cisek and Kalaska (2010) conceptualized the processes of action selection as deciding what to do and action specification as deciding how to do it. Evidence suggests that sensorimotor areas represent multiple potential actions afforded by the environment (and objects within it) simultaneously and selection processes integrate between options based on a number of factors, including stimulus-response compatibility, which according to the current findings, must include sensitivity to the strength of learned associations. Because stimuli are associated with particular responses through experience, the current results suggest that affordance activity in sensorimotor areas come to represent those responses, biasing selection processes in favor of learned associations. Learned affordances of this type offer a fast route from visual stimuli to appropriate motor responses based on previous experience, neural mechanisms that from an evolutionary perspective clearly confer survival advantage. The current findings suggest that, in humans, tools are linked to their most commonly associated action plans at the neural level, stimulusresponse (tool-action) mappings that correspond with motor plans for conventional use.

In explaining the concept of affordance specification at the level of neural activity within sensorimotor areas, we have emphasized the existence of neurons that show visual response selectivity for object properties in agreement with motor response selectivity thought to underlie the specification of corresponding motor parameters (e.g., type of grasp). However, it is important to recognize that action planning is thought to comprise many distinct processes operating in parallel, some of which correspond with specification and implementation of motor parameters, whereas others correspond with more abstract factors, such as overarching goals (Fogassi et al., 2005; Umiltà et al., 2008) and motor attention (Rizzolatti et al., 1987; Andersen and Buneo, 2002; Handy et al., 2003). Affordance activity in sensorimotor areas, including activity showing EXP-RS in the current study, may reflect multiple factors related to action planning.

\section{Task specificity of RS}

Specificity of EXP-RS versus CTR-RS within parietofrontal areas L-AIP, L-vPreC, L-dPreC, and R-SPL (Fig. 2) is thought to reflect differences in the experiential strengths of EXP tool-action versus CTR color-action associations. For the EXP task exclusively, the visuomotor associations that specify the sequence of movements to be performed have the potential to operate on existing associations based on previous knowledge, built up over time with relatively continuous reinforcement, not only through performance of actual actions with tools but also through observation of others using tools. In contrast, the CTR task involved action associations that were newly learned and arbitrary. According to this account, at least some aspects of cortical tool use representations comprise relatively abstract motor programs. This hypothesis coincides well with previous schema theories of tool use and apraxia that have proposed that, as tool use learning unfolds, procedural memory representations that specify the spatiotemporal characteristics of actions are developed and stored within left inferior parietal cortex (Heilman et al., 1982; Rothi et al., 1997). Stored motor schema are thought to be relatively abstract, predicted to specify gross invariant movement features of learned actions, generalizable across different tool exemplars, not specific kinematic or force requirements that would vary according to situational specifics of a given action, such as the particular metrics of a given tool or set of spatial constraints that define an action setting (Buxbaum, 2001). It is possible that specificity for EXP-RS versus CTR-RS reflects retrieval and activation of relatively abstract motor programs for overlearned tool use but not novel color-defined actions.

Differences in ecological validity of associations tied to EXP versus CTR actions may have also been important in driving specificity of EXP-RS. Tools are used to achieve specific goals, real world aims with meaningful outcomes, and recent evidence implicates inferior parietofrontal regions as coding the goals and outcomes of actions (Bonini et al., 2010). Action associations that coincide with ecologically valid behavioral outcomes may be a requisite for lasting encoding within parietofrontal areas. Again, this contrasts with meaningless color-action associations.

Finally, to perform the CTR task, participants may have used specific cognitive strategies to keep arbitrary color-action rules in mind. Activity within L-aINS showing CTR-RS is hypothesized to reflect maintenance of color-defined rules, whereas concurrent CTR-RS in early visual areas B-Calc and R-pCLS is thought to more directly relate with distinguishing handle colors.

\section{Implications and concluding remarks}

A basic fundamental goal of neuroscience is to understand how the brain uses sensory information from the environment to control purposeful behavior. The current study suggests that visual specification of affordances defined as action-relevant object properties within parietofrontal cortex includes access to information based on previous experience. Areas showing RS for the familiar tool use task are thought to reflect transformation of visually defined tool properties to corresponding motor programs for conventional use. Compared with previous neuroimaging studies, the current results provide more selective localization of tool use areas attributable to improved control over potential sensorimotor confounds. Moreover, the findings indicate that visual specification of tool affordances includes information derived from previous experience, instantiated within the same sensorimotor areas underlying their dexterous use. From a theoretical perspective, the advancements made here deviate somewhat from the concept of affordances originally defined by Gibson (1979) and coincide with newer accounts that stress the importance of previous knowledge (Norman, 1999; McGrenere and Ho, 2000). From a neural perspective, the current findings fit within the framework put forth by Cisek and Kalaska (2010) whereby affordance specification is defined as a process that unfolds within sensorimotor neurons involving the specification of potential actions as distinct patterns of activity, considered not only fundamental for action planning and execution but also for resolving competition between multiple potential actions. The current results highlight the importance of previous knowledge. In humans, affordance specification includes access to learned object properties derived from knowledge of function and way of use.

\section{References}

Andersen RA, Buneo CA (2002) Intentional maps in posterior parietal cortex. Annu Rev Neurosci 25:189-220.

Baumann MA, Fluet MC, Scherberger H (2009) Context-specific grasp movement representation in the macaque anterior intraparietal area. J Neurosci 29:6436-6448. 
Begliomini C, Caria A, Grodd W, Castiello U (2007a) Comparing natural and constrained movements: new insights into the visuomotor control of grasping. PLoS One 2:e1108.

Begliomini C, Wall MB, Smith AT, Castiello U (2007b) Differential cortical activity for precision and whole-hand visually guided grasping in humans. Eur J Neurosci 25:1245-1252.

Binkofski F, Dohle C, Posse S, Stephan KM, Hefter H, Seitz RJ, Freund HJ (1998) Human anterior intraparietal area subserves prehension: a combined lesion and functional MRI activation study. Neurology 50:1253-1259.

Bohlhalter S, Hattori N, Wheaton L, Fridman E, Shamim EA, Garraux G, Hallett M (2009) Gesture subtype-dependent left lateralization of praxis planning: an event-related fMRI study. Cereb Cortex 19:1256-1262.

Bonini L, Rozzi S, Serventi FU, Simone L, Ferrari PF, Fogassi L (2010) Ventral premotor and inferior parietal cortices make distinct contribution to action organization and intention understanding. Cereb Cortex 20:1372-1385.

Bub DN, Masson ME, Bukach CM (2003) Gesturing and naming: the use of functional knowledge in object identification. Psychol Sci 14:467-472.

Bub DN, Masson ME, Cree GS (2008) Evocation of functional and volumetric gestural knowledge by objects and words. Cognition 106:27-58.

Buxbaum LJ (2001) Ideomotor apraxia: a call to action. Neurocase 7:445-458.

Buxbaum LJ, Schwartz MF, Carew TG (1997) The role of semantic memory in object use. Cogn Neuropsychol 14:219-254.

Buxbaum LJ, Johnson-Frey SH, Bartlett-Williams M (2005) Deficient internal models for planning hand-object interactions in apraxia. Neuropsychologia 43:917-929.

Carey DP, Harvey M, Milner AD (1996) Visuomotor sensitivity for shape and orientation in a patient with visual form agnosia. Neuropsychologia 34:329-337.

Cavina-Pratesi C, Goodale MA, Culham JC (2007) FMRI reveals a dissociation between grasping and perceiving the size of real 3D objects. PLoS One 2:e424.

Cavina-Pratesi C, Monaco S, Fattori P, Galletti C, McAdam TD, Quinlan DJ, Goodale MA, Culham JC (2010) Functional magnetic resonance imaging reveals the neural substrates of arm transport and grip formation in reach-to-grasp actions in humans. J Neurosci 30:10306-10323.

Chao LL, Martin A (2000) Representation of manipulable man-made objects in the dorsal stream. Neuroimage 12:478-484.

Chouinard PA, Goodale MA (2009) FMRI adaptation during performance of learned arbitrary visuomotor conditional associations. Neuroimage 48:696-706

Cisek P, Kalaska JF (2010) Neural mechanisms for interacting with a world full of action choices. Annu Rev Neurosci 33:269-298.

Creem SH, Proffitt DR (2001) Grasping objects by their handles: a necessary interaction between cognition and action. J Exp Psychol Hum Percept Perform 27:218-228.

Culham JC (2004) Human brain imaging reveals a parietal area specialized for grasping. In: Attention and performance XX: functional brain imaging of human cognition (Kanwisher N, Duncan J, eds). Oxford, UK: Oxford UP.

Culham JC, Danckert SL, DeSouza JF, Gati JS, Menon RS, Goodale MA (2003) Visually guided grasping produces fMRI activation in dorsal but not ventral stream brain areas. Exp Brain Res 153:180-189.

Dinstein I, Hasson U, Rubin N, Heeger DJ (2007) Brain areas selective for both observed and executed movements. J Neurophysiol 98:1415-1427.

Fagg AH, Arbib MA (1998) Modeling parietal-premotor interactions in primate control of grasping. Neural Netw 11:1277-1303.

Fogassi L, Ferrari PF, Gesierich B, Rozzi S, Chersi F, Rizzolatti G (2005) Parietal lobe: from action organization to intention understanding. Science 308:662-667.

Frey SH, Vinton D, Norlund R, Grafton ST (2005) Cortical topography of human anterior intraparietal cortex active during visually guided grasping. Brain Res Cogn Brain Res 23:397-405.

Fridman EA, Immisch I, Hanakawa T, Bohlhalter S, Waldvogel D, Kansaku K, Wheaton L, Wu T, Hallett M (2006) The role of the dorsal stream for gesture production. Neuroimage 29:417-428.

Gallivan JP, McLean DA, Valyear KF, Pettypiece CE, Culham JC (2011) Decoding action intentions from preparatory brain activity in human parieto-frontal networks. J Neurosci 31:9599-9610.

Gibson JJ (1979) The ecological approach to visual perception. Boston: Houghton Mifflin.
Goldenberg G, Spatt J (2009) The neural basis of tool use. Brain 132:1645-1655.

Grèzes J, Tucker M, Armony J, Ellis R, Passingham RE (2003) Objects automatically potentiate action: an fMRI study of implicit processing. Eur J Neurosci 17:2735-2740.

Grill-Spector K, Malach R (2001) fMR-adaptation: a tool for studying the functional properties of human cortical neurons. Acta Psychol (Amst) 107:293-321.

Grill-Spector K, Henson R, Martin A (2006) Repetition and the brain: neural models of stimulus-specific effects. Trends Cogn Sci 10:14-23.

Haaland KY, Harrington DL, Knight RT (2000) Neural representations of skilled movement. Brain 123:2306-2313.

Hamilton AF, Grafton ST (2009) Repetition suppression for performed hand gestures revealed by fMRI. Hum Brain Mapp 30:2898-2906.

Handy TC, Grafton ST, Shroff NM, Ketay S, Gazzaniga MS (2003) Graspable objects grab attention when the potential for action is recognized. Nat Neurosci 6:421-427.

Heilman KM, Rothi LJ, Valenstein E (1982) Two forms of ideomotor apraxia. Neurology 32:342-346.

Hodges JR, Spatt J, Patterson K (1999) "What" and "how": evidence for the dissociation of object knowledge and mechanical problem-solving skills in the human brain. Proc Natl Acad Sci U S A 96:9444-9448.

Hodges JR, Bozeat S, Lambon Ralph MA, Patterson K, Spatt J (2000) The role of conceptual knowledge in object use evidence from semantic dementia. Brain 123:1913-1925.

Jeannerod M, Arbib MA, Rizzolatti G, Sakata H (1995) Grasping objects: the cortical mechanisms of visuomotor transformation. Trends Neurosci 18:314-320.

Johnson-Frey SH (2004) The neural bases of complex tool use in humans. Trends Cogn Sci 8:71-78.

Johnson-Frey SH, Newman-Norlund R, Grafton ST (2005) A distributed left hemisphere network active during planning of everyday tool use skills. Cereb Cortex 15:681-695.

Kriegeskorte N, Simmons WK, Bellgowan PS, Baker CI (2009) Circular analysis in systems neuroscience: the dangers of double dipping. Nat Neurosci 12:535-540.

Króliczak G, Frey SH (2009) A common network in the left cerebral hemisphere represents planning of tool use pantomimes and familiar intransitive gestures at the hand-independent level. Cereb Cortex 19:2396-2410.

Króliczak G, Cavina-Pratesi C, Goodman DA, Culham JC (2007) What does the brain do when you fake it? An FMRI study of pantomimed and real grasping. J Neurophysiol 97:2410-2422.

Króliczak G, McAdam TD, Quinlan DJ, Culham JC (2008) The human dorsal stream adapts to real actions and 3D shape processing: a functional magnetic resonance imaging study. J Neurophysiol 100:2627-2639.

Lauro-Grotto R, Piccini C, Shallice T (1997) Modality-specific operations in semantic dementia. Cortex 33:593-622.

Lewis JW (2006) Cortical networks related to human use of tools. Neuroscientist 12:211-231.

Logothetis NK, Wandell BA (2004) Interpreting the BOLD signal. Annu Rev Physiol 66:735-769.

Logothetis NK, Pauls J, Augath M, Trinath T, Oeltermann A (2001) Neurophysiological investigation of the basis of the fMRI signal. Nature 412:150-157.

Mahon BZ, Milleville SC, Negri GA, Rumiati RI, Caramazza A, Martin A (2007) Action-related properties shape object representations in the ventral stream. Neuron 55:507-520.

Masson ME, Bub DN, Breuer AT (2011) Priming of reach and grasp actions by handled objects. J Exp Psychol Hum Percept Perform 37:1470-1484.

McGrenere J, Ho W (2000) Affordances: clarifying and evolving a concept. Proceedings of Graphics Interface 2000 Conference, Montréal, Québec, Canada, May 15-17.

Milner AD, Goodale MA (1995) The visual brain in action. Oxford, UK: Oxford UP.

Mohr HM, Goebel R, Linden DE (2006) Content- and task-specific dissociations of frontal activity during maintenance and manipulation in visual working memory. J Neurosci 26:4465-4471.

Norman DA (1999) Affordance, conventions, and design. Interactions 6:38-42.

Pilgrim E, Humphreys GW (1991) Impairment of action to visual objects in a case of ideomotor apraxia. Cogn Neuropsychol 8:459-473. 
Raos V, Umiltá MA, Gallese V, Fogassi L (2004) Functional properties of grasping-related neurons in the dorsal premotor area F2 of the macaque monkey. J Neurophysiol 92:1990-2002.

Raos V, Umiltá MA, Murata A, Fogassi L, Gallese V (2006) Functional properties of grasping-related neurons in the ventral premotor area F5 of the macaque monkey. J Neurophysiol 95:709-729.

Riddoch MJ, Humphreys GW, Coltheart M, Funnell E (1988) Semantic systems or system? Neuropsychological evidence reexamined. Cogn Neuropsychol 5:3-25.

Riddoch MJ, Edwards MG, Humphreys GW, West R, Heafield T (1998) Visual affordances direct action: neuropsychological evidence from manual interference. Cogn Neuropsychol 15:645-683.

Rizzolatti G, Craighero L (2004) The mirror-neuron system. Annu Rev Neurosci 27:169-192.

Rizzolatti G, Riggio L, Dascola I, Umiltá C (1987) Reorienting attention across the horizontal and vertical meridians: evidence in favor of a premotor theory of attention. Neuropsychologia 25:31-40.

Rothi LJG, Heilman KM (1997) Apraxia: the neuropsychology of action. East Sussex, UK: Psychology Press.

Rothi LJ, Ochipa C, Heilman KM (1997) A cognitive neuropsychological model of limb praxis and apraxia. In: Apraxia: the neuropsychology of action (Rothi LJG, Heilman KM, eds). East Sussex, UK: Psychology Press.

Rumiati RI, Humphreys GW (1998) Recognition by action: dissociating visual and semantic routes to action in normal observers. J Exp Psychol Hum Percept Perform 24:631-647.

Rumiati RI, Weiss PH, Shallice T, Ottoboni G, Noth J, Zilles K, Fink GR (2004) Neural basis of pantomiming the use of visually presented objects. Neuroimage 21:1224-1231.

Sirigu A, Duhamel JR, Poncet M (1991) The role of sensorimotor experience in object recognition. A case of multimodal agnosia. Brain 114:2555-2573.
Stark A, Zohary E (2008) Parietal mapping of visuomotor transformations during human tool grasping. Cereb Cortex 18:2358-2368.

Taira M, Mine S, Georgopoulos AP, Murata A, Sakata H (1990) Parietal cortex neurons of the monkey related to the visual guidance of hand movement. Exp Brain Res 83:29-36.

Talairach J, Tournoux P (1988) Co-planar stereotaxic atlas of the human brain. New York: Thieme Medical Publishers.

Tolias AS, Smirnakis SM, Augath MA, Trinath T, Logothetis NK (2001) Motion processing in the macaque: revisited with functional magnetic resonance imaging. J Neurosci 21:8594-8601.

Tucker M, Ellis R (1998) On the relations between seen objects and components of potential actions. J Exp Psychol Hum Percept Perform 24:830-846.

Umilta MA, Brochier T, Spinks RL, Lemon RN (2007) Simultaneous recording of macaque premotor and primary motor cortex neuronal populations reveals different functional contributions to visuomotor grasp. J Neurophysiol 98:488-501.

Umiltà MA, Escola L, Intskirveli I, Grammont F, Rochat M, Caruana F, Jezzini A, Gallese V, Rizzolatti G (2008) When pliers become fingers in the monkey motor system. Proc Natl Acad Sci U S A 105:2209-2213.

Valyear KF, Culham JC (2010) Observing learned object-specific functional grasps preferentially activates the ventral stream. J Cogn Neurosci 22:970-984.

Valyear KF, Cavina-Pratesi C, Stiglick AJ, Culham JC (2007) Does toolrelated fMRI activity within the intraparietal sulcus reflect the plan to grasp? Neuroimage 36 [Suppl 2]:T94-T108.

Valyear KF, Chapman CS, Gallivan JP, Mark RS, Culham JC (2011) To use or to move: goal-set modulates priming when grasping real tools. Exp Brain Res 212:125-142.

Yee LT, Roe K, Courtney SM (2010) Selective involvement of superior frontal cortex during working memory for shapes. J Neurophysiol 103:557-563. 